\title{
Vitamine und Gesundheit
}

\author{
Hans Konrad Biesalski
}

\begin{abstract}
Wenn es um gesunde Ernährung und dabei ganz besonders um Vitamine geht, so wird immer wieder darauf hingewiesen: „Wir brauchen keine (zusätzlichen) Vitamine, wir haben genug Obst und Gemüse! “ Beides ist nicht wirklich richtig! Natürlich brauchen wir Vitamine und wir haben durchaus auch genügend Obst und Gemüse. Allerdings sind Obst und Gemüse nicht alleine ausreichend, wenn es um die Versorgung mit Vitaminen geht!
\end{abstract}

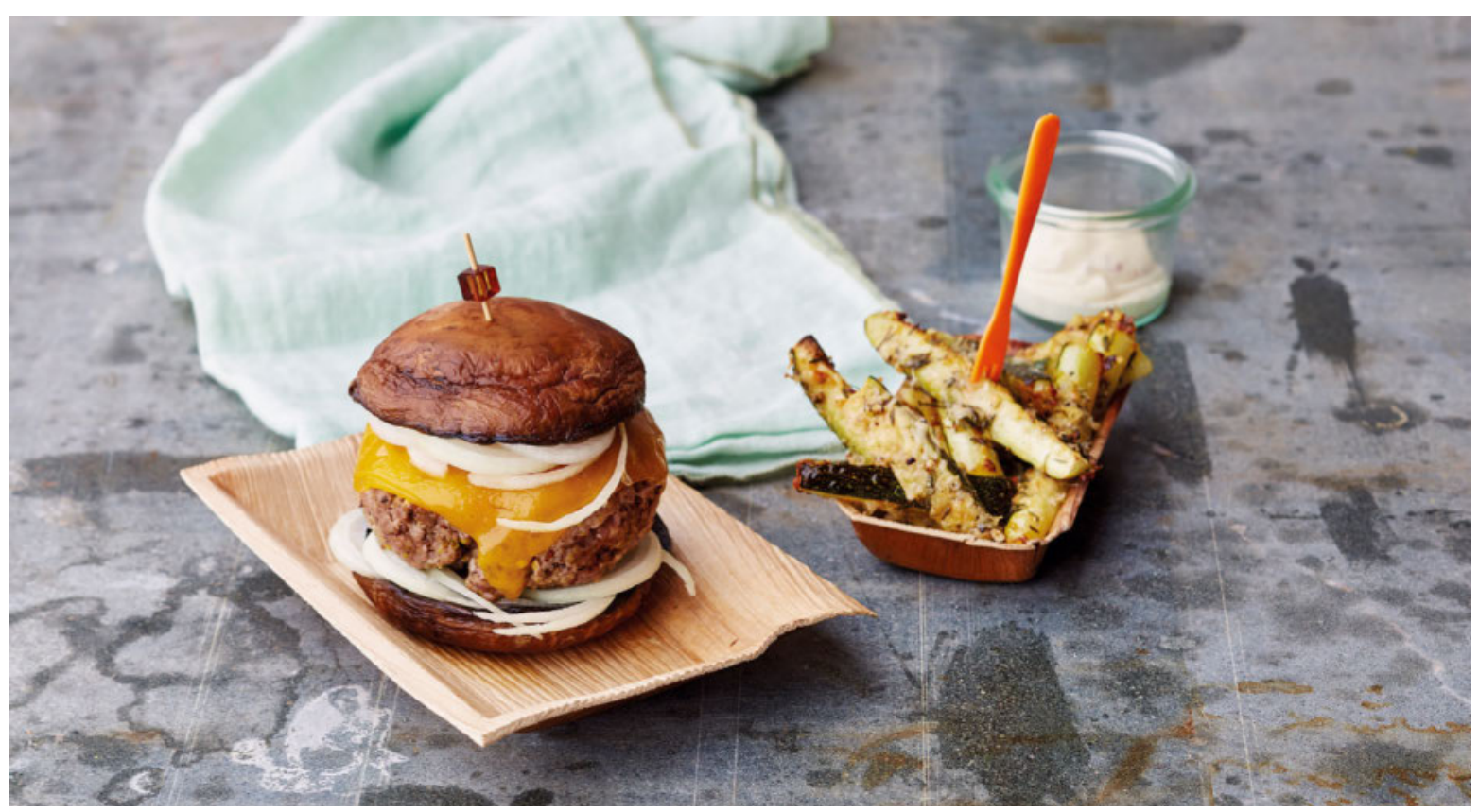

Verborgener Hunger trotz kalorischem Überfluss: Statt eines echten Vitaminmangels, wie z. B. früher bei Skorbut, tritt heute eine Unterversorgung mit diffuseren Symptomen auf. @ Thieme Gruppe/Meike Bergmann

Der Hinweis in der Nationalen Verzehrsstudie, dass in Deutschland kein Vitaminmangel besteht, ist zwar durchaus richtig, aber eben auch nur die halbe Wahrheit. In Deutschland ist das Auftreten eines klinisch sichtbaren Vitaminmangels tatsächlich selten, wenngleich über eine Zunahme an Rachitis bei Kindern berichtet wird.

Der klinisch sichtbare Vitaminmangel stellt ein Endstadium dar, welches sich je nach Vitamin über mehr oder weniger lange Zeit entwickelt hat. Dass bis zu dieser Entwicklung die unzureichende Versorgung mit Vitaminen durchaus Krankheitswert haben kann, wird mangels geeigneter analytischer Verfahren und spezifischer Biomarker oft nicht festgestellt. Um zu verstehen, warum nicht nur die Ernährungswissenschaften, sondern auch die Medizin auf das klinische Bild des Vitaminmangels als ein- zige Indikation einer Intervention ausgerichtet ist, kann man die Geschichte der Vitaminforschung heranziehen.

\section{Geschichtliches}

Das bekannteste Vitamin ist Vitamin C. Denn sein Mangel löste früher eine typische und weit verbreitete und gefürchtete Krankheit, den Skorbut, aus. Vor allem bei Matrosen, deren Ernährung im Wesentlichen aus Schiffszwieback und Dörrfleisch bestand, trat Skorbut als Schreckgespenst auf. Zunächst fing das Zahnfleisch an zu bluten, die Zähne fielen aus, es kam zu Blutungen in den Gelenken und damit zur Arbeitsunfähigkeit und schließlich zum Tod. Teilweise skurrile empirische Beobachtungen führten jedoch dazu, dass entsprechende Rezepte gegen Skorbut entwickelt wurden. 
EMPIRISCHE THERAPIE GEGEN SKORBUT

Zahlreiche zufällige Beobachtungen mündeten in Rezepte gegen die gefürchtete Skorbut-Erkrankung: So verdankt das Skorbutkraut, auch Scharbockskraut genannt, seinen therapeutischen Erfolg der Tatsache, dass 1730 ein skorbutkranker Segler von seinen Kameraden zum Sterben an Land ausgesetzt wurde, dieser sich kriechend von Pflanzen ernährte und gesund wurde und längere Zeit später von seinen Kameraden zu deren Erstaunen wieder auf das Schiff aufgenommen werden konnte. Der Grund für den Erfolg lag darin, dass es sich bei diesem unscheinbaren Hahnenfußgewächs um eine sehr gute Vitamin-C-Quelle gehandelt hat.

Nach und nach wurden mehr Vitamine entdeckt. Es gab dafür diverse Nobelpreise, was die Bedeutung dieser Entdeckungen unterstreicht. Wir müssen uns in die Lage der Menschen versetzen, die plötzlich erlebten, dass häufige schwere und oft zum Tode führende Erkrankungen geheilt werden konnten. So wie die hohe Kindersterblichkeit durch Rachitis, oft in Kombination mit Tuberkulose, oder der gefürchtete Vitamin- $B_{12}$-Mangel, der zu einer Anämie und zur Erkrankung des Rückenmarks führte, um nur 2 Beispiele von vielen zu nennen. Die Beobachtung, dass ein durch eine spezielle Diät verursachter Vitamin- $B_{12}$-Mangel bei Hunden durch den Zusatz von Leber im Futter verhindert bzw. geheilt werden konnte, brachte 3 amerikanischen Wissenschaftlern 1934 den Nobelpreis für Medizin. Die Wissenschaftler wurden ausgezeichnet, da sie eine spezielle Leber-Diät zur erfolgreichen Behandlung dieser schweren Erkrankung entwickelt hatten. Dass der entscheidende Wirkstoff Vitamin $\mathrm{B}_{12}$ war, wurde erst 14 Jahre später beschrieben.

Es war ebenfalls eine empirische Beobachtung, dass Kinder mit Rachitis, die oft mit Tuberkulose einherging, nicht nur einen milderen Verlauf der Tuberkulose hatten und überlebten, sondern sich auch die rachitischen Symptome teilweise zurückbildeten, wenn sie in die Sonne gesetzt wurden. Die Ursache lag darin, dass das durch Sonnenlicht in der Haut gebildete Vitamin D nicht nur die Rachitis als Vitamin-D-Mangelerkrankung heilte, sondern dass Vitamin D auch die Bildung eines körpereigenen Antibiotikums (Defensin: Cathelicidin) in der Lunge induzierte, welches die Tuberkulose bekämpfte. Letzteres wissen wir erst seit 10 Jahren. Dennoch behandelte man bereits 1915 die Tuberkulose wie auch die Rachitis bei Kindern mithilfe der Höhensonne.

Merke

Bis heute bestimmt der Mangel an einem einzelnen Vitamin die Indikation zur Behandlung.
Die Entdeckung, dass sich viele schwere und oft zum Tode führende Erkrankungen durch die Gabe eines einzelnen synthetisch hergestellten Vitamins heilen ließen, hat den Umgang mit Vitaminen bis heute geprägt. Erst wenn das Defizit klinisch oder durch Laboranalyse nachgewiesen ist, besteht - so die häufige Aussage - eine Indikation zur Behandlung. Solange die empfohlenen Zufuhrwerte erreicht werden, besteht folglich auch kein Grund, über einen Mangel nachzudenken.

\section{Bedarf und Empfehlung}

Mit der Entdeckung der Wirkung und der chemischen Struktur der Vitamine konnten nicht nur viele Mangelkrankheiten geheilt werden, sondern es stellte sich auch bald die Frage: Wie lässt sich ein solcher Vitaminmangel durch Ernährung verhindern bzw. wie viel braucht der Mensch, um ausreichend versorgt zu sein?

Was genau ist der Bedarf, d. h. die Menge eines Vitamins, mit der ein Mangel verhindert werden kann? Der sogenannte geschätzte mittlere Bedarf (Estimated Average Requirement, EAR) ist eine grobe Schätzung, die als Mittelwert die Aufnahme eines Vitamins bei einer gesunden erwachsenen Bevölkerung ermittelte. Innerhalb der so dargestellten Normalverteilung haben diejenigen ein steigendes Risiko für die Entwicklung einer Mangelerkrankung, die unterhalb des Mittelwertes liegen, während die, die oberhalb liegen, ein abnehmendes Risiko aufweisen.

Bei den etablierten EAR-Werten muss allerdings berücksichtigt werden, dass sich die Ernährung der Menschen vor 30-40 Jahren von der heutigen in vielen Facetten unterschied. Es gab weder Convenience-Food noch Tiefkühlkost in der Menge, wie wir sie heute vorfinden.

\section{Merke \\ Die meisten EAR-Werte wurden vor 30 und mehr Jahren bestimmt und bis heute nicht wesentlich über- arbeitet.}

Nimmt man 2 Standardabweichungen des Mittelwertes zu diesem hinzu, so gelangt man zu dem Wert, den wir heute als Empfehlung nehmen. Mit diesem Wert sollten dann 97,5\% der Bevölkerung ausreichend versorgt sein. Diese Empfehlungen haben sich seither nur wenig verändert. Manche Werte, wie die für Folsäure, hat man nach unten korrigiert, andere dagegen, wie die für Vitamin D, erheblich nach oben. Streng genommen sind die Empfehlungen nur als Orientierungshilfe für die Gemeinschaftsverpflegung geeignet. Was der Einzelne braucht, in welchem Lebensabschnitt wie viel und ganz besonders bei Krankheiten, ist mehr oder weniger unbekannt. Für den individuellen Bedarf haben wir keine Möglichkeit, diesen auch nur näherungsweise zu berechnen. 


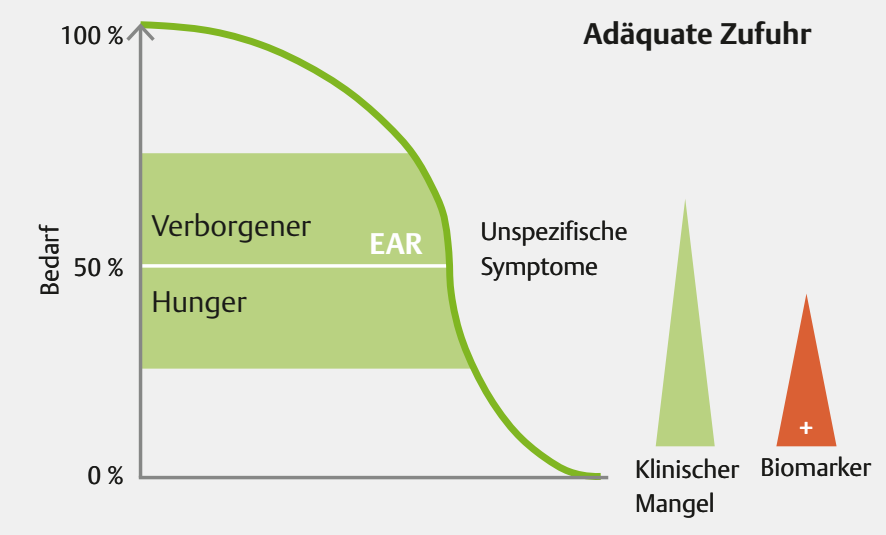

Abb. 1 Bedarfsdeckung und Mangelentwicklung.

Wird der Bedarf gedeckt, d. h. ist die Zufuhr mit der Ernährung ausreichend, besteht keine Notwendigkeit, zusätzlich Vitamine zuzuführen. Dabei kann die Vitaminzufuhr durchaus immer wieder für begrenzte Zeit (je nach Halbwertszeit des Vitamins) unter $100 \%$ der Bedarfsdeckung liegen, ohne dass damit das Risiko einer Unterversorgung besteht. Je mehr sich die mittlere Zufuhr dem EAR-Wert nähert, desto eher kann es zur Entwicklung unspezifischer Symptome kommen, die jedoch selten mit dem Fehlen des Vitamins in Verbindung gebracht werden ( $\mathbf{A} \mathbf{A b}$. 1). Dieser auch als „verborgener Hunger“ bezeichnete Zustand stellt ein weltweites Problem dar und betrifft vor allem die Vitamine A und D sowie Eisen und Zink. Hier besteht inzwischen Einigkeit, dass diese unzureichende Versorgung einen deutlichen Einfluss auf die Gesundheit und die Entwicklung der Betroffenen hat. Erst sehr viel später kommt es zu den Zeichen des klinischen Mangels, der dann auch durch entsprechende Biomarker analytisch erfasst werden kann.

\section{Merke}

Vor Auftreten klinisch manifester Mangelsymptome besteht bereits eine unzureichende Versorgung mit negativen Auswirkungen auf die Gesundheit.

\section{Analytik}

Die Pellagra-Epidemie in den USA zu Beginn des 20. Jahrhunderts war Folge von Armut und resultierender einseitiger Ernährung: Mais und wenig Fleisch [1]. Mais enthält sehr wenig Niacin und kaum Tryptophan. Der Phänotyp der Pellagra äußert sich in einer abnehmenden kognitiven Leistung, Störungen von Sprache und der Fähigkeit, zu rechnen (Akalkulie), und geht im Verlauf mit einer progressiven Demenz und unterschiedlichen neuro-psychiatrischen Erkrankungen einher. Erst 1937 wurde Niacin erstmals beschrieben und die Erkrankung konnte behandelt werden. Allerdings wurde und wird die Pellagra bis heute meistens nicht beachtet. Denn oft werden die frühen Veränderungen übersehen: eine Dermatitis an Hautstellen, die der Sonne ausgesetzt sind - dies hat zur Bezeichnung Pellagra sine Pellagra geführt. Eine Bestimmung des Status im Blut oder Urin ist mit entsprechendem technischen Aufwand (GC-MS) zwar möglich, jedoch für die Diagnostik oft nicht aussagekräftig.

In den wenigsten Fällen sind Blutwerte geeignet, eine inadäquate Versorgung frühzeitig zu entlarven. Bis zu einem gewissen Grad ist dies bei wasserlöslichen Vitaminen ( $B_{12}$, $B_{6}$ ) möglich, bei fettlöslichen jedoch so gut wie gar nicht mit der Ausnahme von Vitamin D.

Der Vitamin-A-Mangel als ein weltweit verbreitetes Problem (etwa 200 Millionen Betroffene, vorwiegend Frauen und Kinder) führt bereits lange vor Auftreten der ersten klinischen Symptome (Nachtblindheit) zu einer erhöhten Sterblichkeit bei Kindern und einer Eisen-refraktären Anämie. Vitamin C verteilt sich auf die unterschiedlichsten Kompartimente und je nach Analyse und Gesundheitszustand des Probanden können hier sehr verschiedene Werte gemessen werden.

\section{Merke}

Blut stellt das Transportsystem der Vitamine zu den Zellen dar und ist daher nur bedingt geeignet, eine Aussage über die Versorgung zu machen.

Exemplarisches Beispiel ist hier Vitamin D. In der Annahme, dass die Bildung des aktiven Metaboliten (Hydroxylierung des 25-(OH)-D in Position 1 zu 1,25-Dihydroxycholecalciferol) ausschließlich in der Niere erfolgt, hat man Statusanalysen durch Bestimmung dieses Metaboliten vorgenommen. Die Ergebnisse solcher Analysen deckten sich nicht mit verschiedenen klinischen Bildern, die man einer Vitamin-D-Unterversorgung zuschrieb. Erst als klar wurde, dass die Aktivierung des 25-(OH)-D auch in vielen anderen Geweben möglich ist, ließ sich durch Bestimmung des 25$(\mathrm{OH})$-D im Blut ein (bisher) verwertbarer Indikator für den Vitamin-D-Status finden. Eine ganze Reihe von bisher nicht bekannten Beziehungen zu Krankheitszuständen konnte so mit einem niedrigen Vitamin-D-Status in Beziehung gebracht werden. In der Konsequenz setzte man nun die Empfehlungen um das 4-Fache (von 5 auf $20 \mu \mathrm{g} / \mathrm{Tag}$ ) nach oben. Bis dahin schienen $5 \mu \mathrm{g} /$ Tag ausreichend, ein Wert, der in der Mitte des letzten Jahrhunderts als ausreichend für die Prävention der Rachitis ermittelt wurde.

Für die meisten anderen Vitamine steht eine solche valide Statusbestimmung noch aus. Wie aber lässt sich dann eine unzureichende Versorgung erfassen und gegebenenfalls behandeln? 


\section{Verborgene Wirkung - verborgener Hunger}

Es gibt verschiedene Risikogruppen von Personen, die mit einem oder mehreren Mikronährstoffen häufiger unterversorgt sind. Einerseits sind dies Menschen mit speziellen einseitigen Ernährungsstilen (vegan, häufige Reduktionsdiäten, Essstörungen u. a.), bei anderen spielen Alter und Lebenssituation (z. B. Schwangerschaft, frühkindliche Entwicklung, Einkommen und Bildung) eine wichtige Rolle. Klinisch sichtbaren Mangel wird man hier nur in den seltensten Fällen finden, vielmehr eine Situation, die die WHO als verborgenen Hunger definiert.

DEFINITION „VERBORGENER HUNGER“

Verborgener Hunger ist eine unzureichende Versorgung mit einem oder mehreren Mikronährstoffen ohne klinisch eindeutige Symptomatik.

Der verborgene Hunger stellt ein weltweites Problem dar und betrifft am häufigsten die folgenden Mikronährstoffe:

- Vitamin A

- Folsäure

- Vitamin D

- Eisen

- Zink [2].

\section{Merke}

Die häufigste Ursache für verborgenen Hunger ist Armut, die sich auch in Deutschland zunehmend ausbreitet.

Betroffen vom verborgenen Hunger infolge Armut in Deutschland sind vor allem alleinerziehende Mütter und ihre Kinder (20\% aller Kinder in Deutschland) sowie alte Menschen. Zu Recht spricht man heute von Ernährungsarmut. Bei jungen Frauen hat der verborgene Hunger Konsequenzen besonders in der Schwangerschaft. Bei Kindern sind in den ersten Lebensjahren durch eine unzureichende Versorgung mit Mikronährstoffen körperliches Wachstum, Gesundheit und kognitive Entwicklung nachhaltig gestört [3].

\section{Versorgung in der Schwangerschaft}

\section{Folsäure}

In der Schwangerschaft und Stillzeit wird für verschiedene Vitamine und Minerale zwar ein Mehrbedarf angegeben, dieser ist jedoch nicht Ergebnis wissenschaftlicher Studien, sondern wird mehr oder weniger geschätzt. Ein exemplarisches Beispiel, dass diese Schätzung nicht ausreichend war, ist die Folsäure. Hier hat man erst vor etwa 20 Jahren erkannt, dass eine Versorgung mit Folsäure, wie sie bis dahin durch die Empfehlungen gegeben wurde, nicht ausreicht, um Neuralrohrdefekte zu verhindern. Erst die Empfehlungen für junge Frauen mit Kinderwunsch, dass sie Folsäure substituieren sollen, sowie die in einigen Ländern erfolgte Anreicherung von Mehl und anderen Lebensmitteln mit Folsäure hat die Zahl der Neuralrohrdefekte je nach Land teilweise drastisch reduziert. Es hat allerdings viele Jahre gedauert, bis sich durchgesetzt hatte, dass die bis dahin gegebenen Empfehlungen für Folsäure für die Schwangerschaft nicht ausreichend waren.

\section{Biotin}

In der Zwischenzeit geht man einem ähnlichen Phänomen für das Vitamin Biotin nach. Hier mehren sich Stimmen, dass eine gute bis sehr gute Versorgung mit Biotin in der Schwangerschaft dazu beitragen kann, unterschiedliche Missbildungen zu verhindern. Die fetale und embryonale Blutkonzentration an Biotin ist um das 3- bis 17-Fache höher als die Konzentrationen im Blut der Mutter [4], [5], [6]. Für den Biotin-Bedarf bestehen mangels entsprechender Daten bisher noch keine Empfehlungen, sondern lediglich sogenannte Schätzwerte. Da eine Unterversorgung mit Biotin wegen des hohen Bedarfs in der Schwangerschaft nicht ausgeschlossen ist und gleichzeitig mit dem in Tierexperimenten gezeigten Risiko für Missbildungen einhergeht, sollte ein solcher Zusammenhang wissenschaftlich intensiver untersucht werden. Nur so kann man daraus entsprechende Empfehlungen ableiten.

Merke

Bisher gibt es keine validen Richtwerte, sondern lediglich Schätzwerte für den Biotin-Bedarf in der Schwangerschaft.

\section{Multivitamin-Mineral-Supplementation}

Die Ergebnisse verschiedener großer Metaanalysen legen nahe, dass bei Schwangeren nicht nur wie bisher Folsäure und Eisen zur Substitution empfohlen werden sollten, sondern ein Multivitamin-Mineral-Supplement mit Folsäure und Eisen. Diese Metaanalysen haben gezeigt, dass ein Multivitamin-Mineral-Supplement zu einer deutlicheren Senkung des Risikos für geringes Geburtsgewicht oder Missbildungen führt, als dies bisher durch die Folsäure-Eisen-Supplementierung gegeben war. Welcher der Mikronährstoffe in den Supplementen letztlich dafür verantwortlich ist, oder ob es die Summe aller Mikronährstoffe ist, muss noch geklärt werden.

\section{FAZIT FÜR SCHWANGERSCHAFT}

Die Daten der Nationalen Verzehrsstudie zeigen, dass gerade bei Frauen im gebärfähigen Alter die empfohlenen Zufuhrwerte bei einer Reihe von Mikronährstoffen nicht erreicht werden. Dies mag im Falle gesunder Frauen ohne größere Bedeutung sein, im Falle einer Schwangerschaft kann dies jedoch für die kindliche Entwicklung ein Risiko darstellen. 


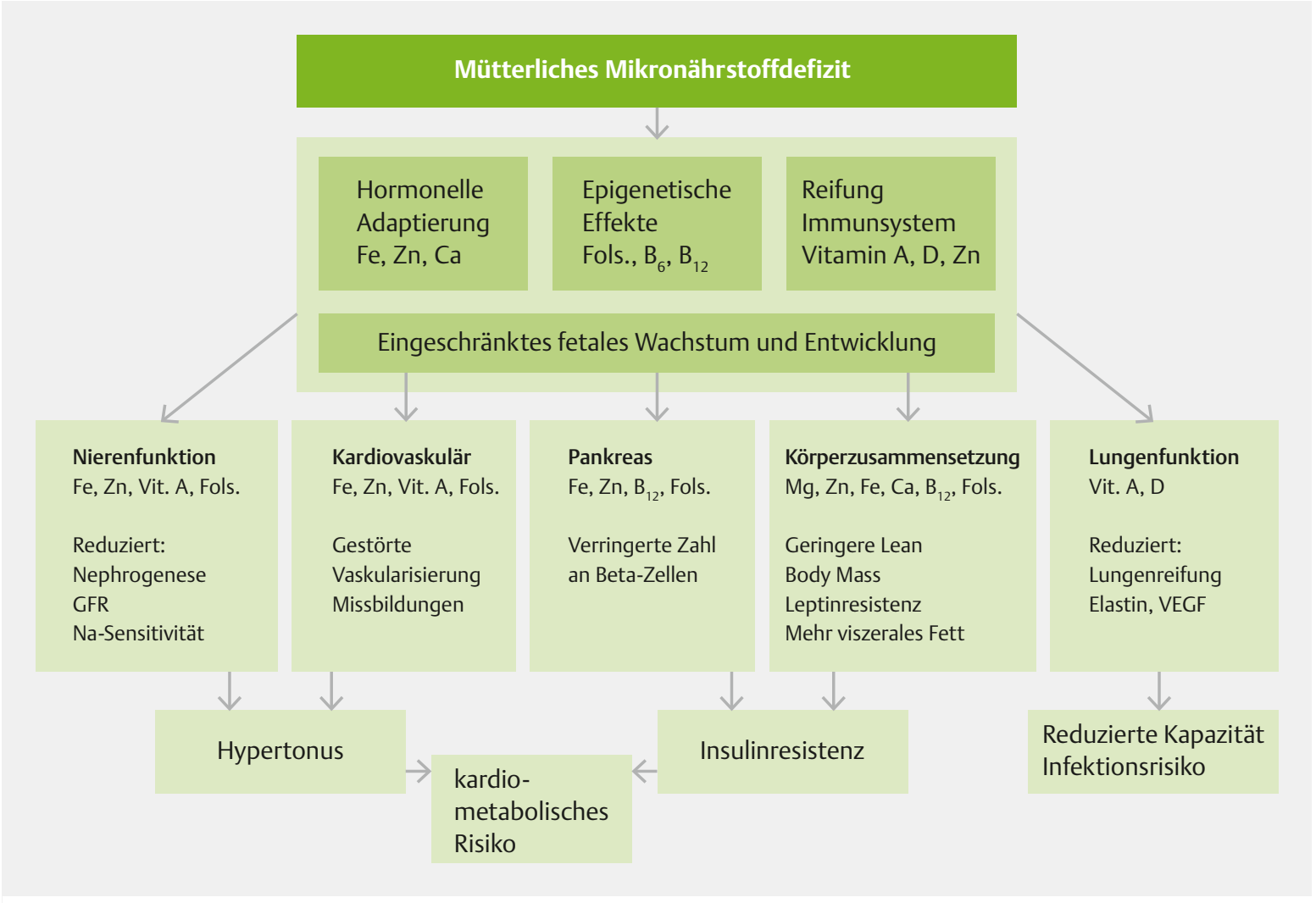

Abb. 2 Angenommene Beziehungen zwischen nicht übertragbaren Erkrankungen und Mikronährstoffversorgung in der Schwangerschaft. Die Unterversorgung kann dabei sowohl durch eine inadäquate Ernährung als auch durch eine Plazentainsuffizienz verursacht sein.

\section{Auswirkungen auf die spätere Entwicklung}

Für die Phase der Schwangerschaft geht es nicht nur um die Vermeidung von Missbildungen, sondern auch um die spätere Entwicklung des Kindes. Denn inzwischen liegen zunehmend wissenschaftliche Daten vor, die zeigen, dass eine unzureichende Vitaminversorgung in der Schwangerschaft Einfluss auf die spätere Entwicklung des Kindes bis in das Erwachsenenalter haben kann. Solche epigenetischen Effekte werden seit Kurzem für die Entwicklung der sogenannten nicht kommunizierbaren Erkrankungen mit verantwortlich gemacht ( $\triangleright$ Abb. 2).

Oft ist es das geringe Geburtsgewicht, welches eine Mangelversorgung signalisiert mit möglichen Konsequenzen für die Organentwicklung und deren Funktion. Diese als DOHaD-Hypothese (Developmental Origin of Health and Disease) bezeichneten Zusammenhänge beschreiben die Bedeutung verschiedenster Umweltfaktoren in der Schwangerschaft und den ersten Lebensjahren des Kindes für die spätere gesundheitliche Entwicklung. Zu diesen Umweltfaktoren gehört auch die Ernährung. In diesem Zusammenhang spielen Mikronährstoffe eine ganz wesentliche Rolle.

\section{Merke \\ Die Bedeutung der Versorgung einzelner Mikronähr- stoffe für die Entwicklung des Kindes ist bisher erst in Ansätzen verstanden. \\ Mangelernährung im Kindesalter und Konsequenzen}

\section{Auswirkungen des Mangels}

Die Konsequenz der Unterversorgung in der Schwangerschaft besonders bei der armen Bevölkerung zeigt sich exemplarisch in der körperlichen wie kognitiven Entwicklung der Kinder. Das Adipositas-Risiko, so die KiGGS [7] und andere Studien, ist bei niedrigem Sozialniveau bei Kindern 3-mal höher als bei gutem Einkommen. Kinder aus armen Verhältnissen in Brandenburg werden tendenziell kleiner (bis zu 1,8 cm) und zeigen bei den Einschulungsuntersuchungen deutlich häufiger Sprachentwicklungsstörungen und Beeinträchtigung der geistigen Entwicklung (17,1 \% bzw. 12,7\%) im Vergleich zu Kindern aus Familien mit hohem Sozialstatus (5,2\% bzw. 0,8\%) [8]. 


\section{STUNTING}

Eine Abweichung der Körpergröße um 2 Standardabweichungen vom Mittelwert wird als Stunting bezeichnet. Stunting ist der Phänotyp der Mangelernährung.

Im Falle der Brandenburger Kinder beträgt die Abweichung vom Mittelwert 1,2 Standardabweichungen.

Folgt man den Beobachtungen von Kimberly Noble, so zeigt sich bei niedrigem Sozialstatus im Vergleich zu hohem Status bei den betroffenen Kindern ein geringeres Hippocampusvolumen sowie eine Sprachentwicklungsstörung bei gleichem Wortschatz [9].

Der Hippocampus ist der Hirnanteil, welcher für das Kurzzeitgedächtnis und vor allem den Spracherwerb von besonderer Bedeutung ist. Bemerkenswert ist, dass für die kognitive Entwicklung vor allem Jod, Eisen und Vitamin $D$ besonders wichtig sind. In einer der wenigen Untersuchungen bei Kleinkindern (10-36 Monate) in Deutschland waren dies genau die Mikronährstoffe, die deutlich unter den Empfehlungen lagen [10].

\section{Ernährungssituation verbessern}

Zweifellos ist die Entwicklungsstörung bei Kindern, die in Armut leben, nicht nur durch eine unzureichende Ernährung zu erklären. Es ist jedoch schwer zu verstehen, warum der Hartz-IV-Satz für die Ernährung von Kindern teilweise deutlich unter dem liegt, was für eine gesunde Ernährung ausgegeben werden muss [11]. Preisgünstige Lebensmittel, zu denen Nudeln, Reis, Kartoffeln, aber auch oft fettes Fleisch und billige Wurstwaren gehören, haben einen hohen Energieanteil, dafür aber eine geringe Dichte an Mikronährstoffen [12]. Die Mangelernährung ist hier vorprogrammiert.

Merke

Eine Verbesserung der Ernährung bei Kindern in Armutsverhältnissen ist weitaus leichter zu erreichen als Eingriffe in das soziale Gefüge.

Bei entsprechendem politischem Willen, wie z. B. eine kostenlose Ernährung in Kindertagesstätten und Ganztagsschulen, wie sie in skandinavischen Ländern seit vielen Jahren praktiziert wird, ließe sich nicht nur eine Verbesserung der Ernährungssituation bei den betroffenen Kindern erreichen, sondern auch eine nicht unerhebliche Kostendämpfung für die Behandlung des kindlichen Übergewichtes und seiner Folgen. 


\section{Fazit}

Die Bedeutung einer unzureichenden Ernährung für die kindliche Entwicklung und die spätere Leistungsfähigkeit sollte nicht länger dem Zufall überlassen bleiben. Ernährungsprogramme und Empfehlungen bringen wenig, wenn die empfohlene Ernährung für den Einzelnen nicht finanzierbar ist. Dabei darf nicht übersehen werden, dass übergewichtige Kinder auch 10-mal häufiger im Erwachsenenalter übergewichtig sind.

\section{KERNAUSSAGEN}

Wir verstehen nach und nach, dass Vitaminwirkungen keinesfalls nur über die beobachteten Mangelsymptome zu erklären sind, sondern sich sehr viel komplexer darstellen als bisher vermutet. Zunehmend wird aber deutlich, dass Menschen, die in Armut leben, Schwangere, Kleinkinder und alte Menschen ein erhöhtes Risiko für eine Unterversorgung aufweisen. Das häufig gebrauchte Argument, jeder könne sich gesund ernähren und wir hätten in Deutschland keinen Mangel, ist wenig hilfreich. Zweifellos lässt sich eine Unterversorgung an Mikronährstoffen in Deutschland durch eine ausgewogene Ernährung verhindern. Vorausgesetzt sind dabei grundlegende Kenntnisse und ein ausreichendes Einkommen [13].

\section{Über den Autor}

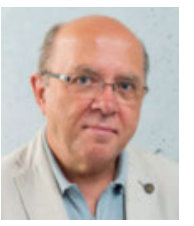

\section{Prof. Dr. Hans Konrad Biesalski}

Prof. Hans Konrad Biesalski ist Ernährungsmediziner und befasst sich seit 30 Jahren mit Vitaminen und deren Auswirkungen auf den Menschen.

\section{Korrespondenzadresse}

\section{Prof. Dr. Hans Konrad Biesalski}

Institut für Biologische Chemie und Ernährungswissenschaft Universität Hohenheim

Garbenstr. 30

70593 Stuttgart

biesal@uni-hohenheim.de

\section{Literatur}

[1] Roe D. A Plague of Corn: The social history of Pellagra. London: Cornell Univ Press; 1973

[2] Biesalski HK. Verborgener Hunger. Heidelberg: Springer; 2013

[3] Johnson SB, Riis JL, Noble KG. State of the art review: Poverty and the developing brain. Pediatrics 2016; 137: e20153075

[4] Mock DM. Biotin: From nutrition to therapeutics. J Nutr 2017; 147: 1487-1492

[5] Mock DM. Marginal biotin deficiency is common in normal human pregnancy and is highly teratogenic in mice. J Nutr 2009; 139: 154-157

[6] Zempleni ], Mock DM. Marginal biotin deficiency is teratogenic. Proc Soc Exp Biol Med 2000; 223: 14-21

[7] Ergebnisse der Kinder- und Jugendgesundheitsstudie KiGGS - Zusammenfassung von Beiträgen im Bundesgesundheitsblatt Mai/Juni 2007. Im Internet: https://www.kiggs-studie. de/fileadmin/KiGGS-Dokumente/KiGGS1_Zusammenfassung_20140623.pdf (Stand: 03.03.2018)

[8] Baten J, Böhm A. Children's height and parental unemployment: a large-scale anthropometric study on Eastern Germany, 1994-2006. German Economic Review 2009; 11: 1-24

[9] Noble KG, Houston SM, Brito NH et al. Family income, parental education and brain structure in children and adolescents. Nat Neurosci 2015; 18: 773-778

[10] Hilbig A, Drossard C, Kersting M, Alexy U. Nutrient adequacy and associated factors in a nationwide sample of German toddlers. J Pediatr Gastroenterol Nutr 2015; 60: 1-8

[11] Kersting M, Clausen K. Wie teuer ist eine gesunde Ernährung für Kinder und Jugendliche? Die Lebensmittelkosten der Optimierten Mischkost als Referenz für sozialpolitische Regelleistungen. Ernährungs-Umschau 2007; 54: 508-513

[12] Dwyer JT, Drewnowsky A. Food and nutrition security. In: Biesalski HK, Drewsnowski A, Dwyer JT et al., eds. Sustainable nutrition in a changing world. Heidelberg: Springer; 2017

[13] Biesalski HK. Vitamine und Minerale. Stuttgart: Thieme; 2016

\section{Bibliografie}

DOI https://doi.org/10.1055/s-0044-100982

Online-Publikation: 29.5.2018

Ernährung \& Medizin 2018; 33: 74-80

(c) Georg Thieme Verlag KG Stuttgart · New York ISSN 1439-1635 